\title{
Research on the Impacts of BIM on Information Exchange between Stakeholders in Construction Project
}

\author{
Yingnan Yang ${ }^{1, a}$, Yanfei Jiang ${ }^{1, b *}$ \\ ${ }^{1}$ College of Civil Engineering and Architecture, Zhejiang University, China \\ ayyn@zju.edu.cn; b21412007@zju.edu.cn
}

Keywords: BIM; Information Exchange; Impact; Stakeholder; Project

\begin{abstract}
In order to analyze the impacts of BIM on information exchange between construction project stakeholders, this paper studied from three essential information exchange modes. At last, the simplified version of Shannon-Weaver model II was chosen as the analysis framework. The whole process of information exchange was gradually analyzed with the help of literature, conclusions were given as follows: because of the adoption of BIM, the supplier and demander of information may change; richer, more clear and accurate information were created; the efficiency of information transmission was improved and the possibility of wrong interpretation of information was reduced. Apart from this, these impacts may produce a series of effects, so project managers should consider about these impacts when they make decisions. Also, with the special analysis of the impacts, this paper can provide some advices for decision makers when they decide if they should adopt BIM even lacking of empirical support.
\end{abstract}

\section{Introduction}

From beginning to the end, construction project will experience a long life cycle. It is not only a material production process, but also a process of information production, collection, transmission, storage and application[1]. Also, it will involve clients, designers, contractors, supervisions, the government and other stakeholders. While, information exchange between stakeholders is often the critical factors affecting the success of project. Ahmad Rtishad once said, "The exchange of data and information is essential for project management in construction projects". And the Project Management Institute of the United States puts the management of information exchange as one important part of the body of project management knowledge. However, the issue of information exchange between project stakeholders is always serious. According to records, almost two-thirds of questions existed in the implementation of construction projects is related to information exchange; ten to thirty-three percent of the increased cost is related to information exchange questions[1].

BIM has brought many benefits from its appearance in construction industry, its influence on information exchange is one of those benefits, and the decrease of RFI has proved that[2]. In order to study the influence of BIM on information exchange, this paper began with the model of information exchange, gradually analyzed the impact of BIM on the process of information exchange between stakeholders.

\section{Modes of Information Exchange}

Generally speaking, Information exchange refers to the information transmission and feedback through various means and channels. Specifically, information exchange is a kind of social activity which intends to realize information transmission and exchange between the sender and the receiver by the help of some symbolic system and transmission channel[3]. The process of that includes information sending, information transmission, information receiving and feedback. Information exchange is the important content of information science and information management science. There are many information exchange modes up to now, this paper mainly study three simple common modes for the request of research.

Harold Lasswell's 5W model[4]. As shown in Fig. 1, in his paper < The Structure and Function 
of Communication in Society >, Harold Lasswell proposed a $5 \mathrm{~W}$ model of information transmission, which established the basic framework of communication research. $5 \mathrm{~W}$ specifically refer to who, what, what channel, whom and what effect.

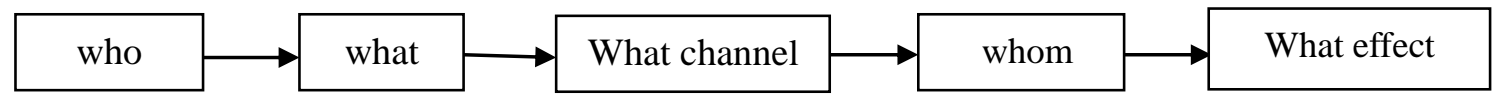

Figure 1. Harold Lasswell's 5W model

Shannon- Weaver model I[3]. In 1949, Shannon and his partner Weaver proposed a communication system model. Later, the model became the basic model of information theory, as shown in Fig. 2. In this model, information source sends out information, after the transmitter, information is turned into a signal. When the signal is transmitted in the channel, it is interfered by the "noise". Then the signal will be turned back into information after the receiver. Finally, information is transferred to destination.

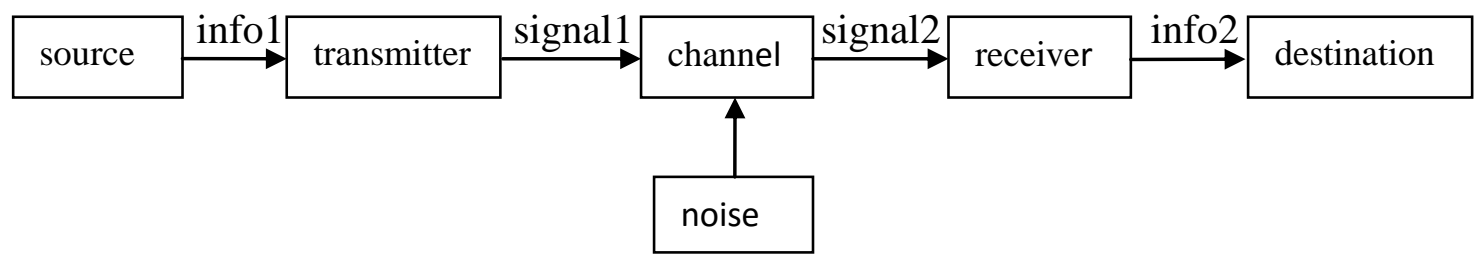

Figure 2. Shannon- Weaver model I

Schramm Model1[5]. In 1995, Schramm proposed three modes of information exchange, one of them was discussed in this paper, as shown in Fig. 3. This model is similar to Shannon- Weaver model I.

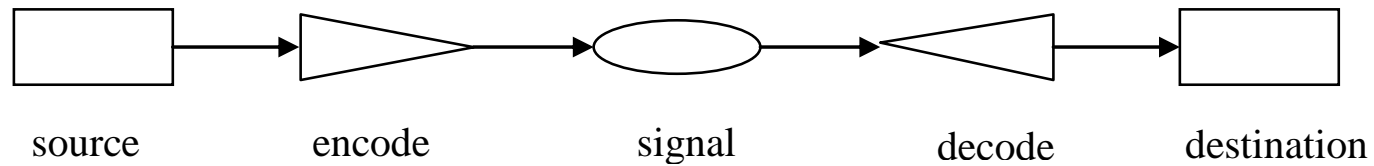

Figure 3. Schramm Model I

In addition to the above three models, by further consideration of the specific circumstances the scholars had proposed Shannon-Weaver model II, Schramm Model II, VIC Model, Mikhailovmodel, Lancaster model and other more models.

\section{Comparative Analysis of the Three Modes of Information Exchange}

Though almost every aspect is included in Lasswell's 5W model, while, it is just a one-way linear model without noticing the effect of feedback. Compared with Lasswell's 5W model, ShannonWeaver model I proposed the concept of noise in the information exchange, suggesting that information will be disturbed in transmission process. While, there is the same flaw with Lasswell's $5 \mathrm{~W}$ model in the model. That is, it's still just a one-way linear transmission without feedback. Schramm model I and Shannon- Weaver model I is very similar, besides the same flaw with Shannon- Weaver model I, it doesn't have the process of noise. In the end, we adopted the ShannonWeaver model I and some necessary complement, i.e. the feedback of information, as the basis of the analysis which aimed to study the influence of BIM on construction project stakeholders' information exchange. As shown in Fig. 4, it is the simplified version of Shannon- Weaver model II in fact. 


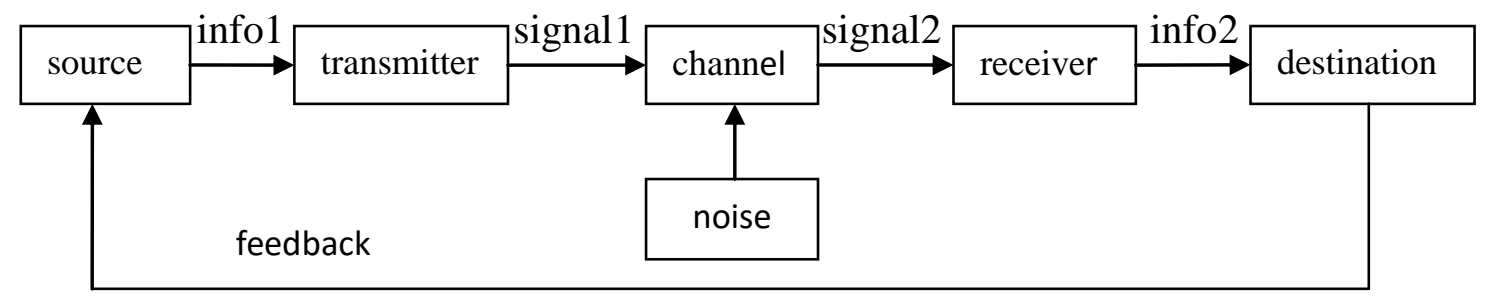

Figure 4. The simplified version of Shannon- Weaver model II

\section{Information Exchange between Stakeholders in Traditional Project}

Construction project information refers to the data, information, reports and documents in project, including the organizational information, technical information, economic information and management information, etc. and the features of huge amount, complex types, various sources, scattered storage and dynamic changes, etc[6]. Information exchange between project stakeholders refers to stakeholders' information exchange and sharing and is the basis of coordination and cooperation. while, there are many questions such as information waste, information loss, low efficiency of information exchange and other more questions because of the fragmented industry structure and the paper media of information transmission[1], which hinders the improvement of the efficiency of construction industry.

\section{Impacts of BIM on the Information Exchange between Construction Project Stakeholders}

Impacts of BIM on information source. Information source refers to the one who sends out information, the owner of information and the source of information for information sink. Information source include clients, designers, contractors, consultants, supervisions etc, meaning every stakeholders in traditional construction project. For the project which has adopted BIM, a large number of software are involved. So according to the concept of stakeholders, software vendors become the new stakeholders in BIM project, as well the new information source, and meaning that the adoption of BIM makes information source increased.

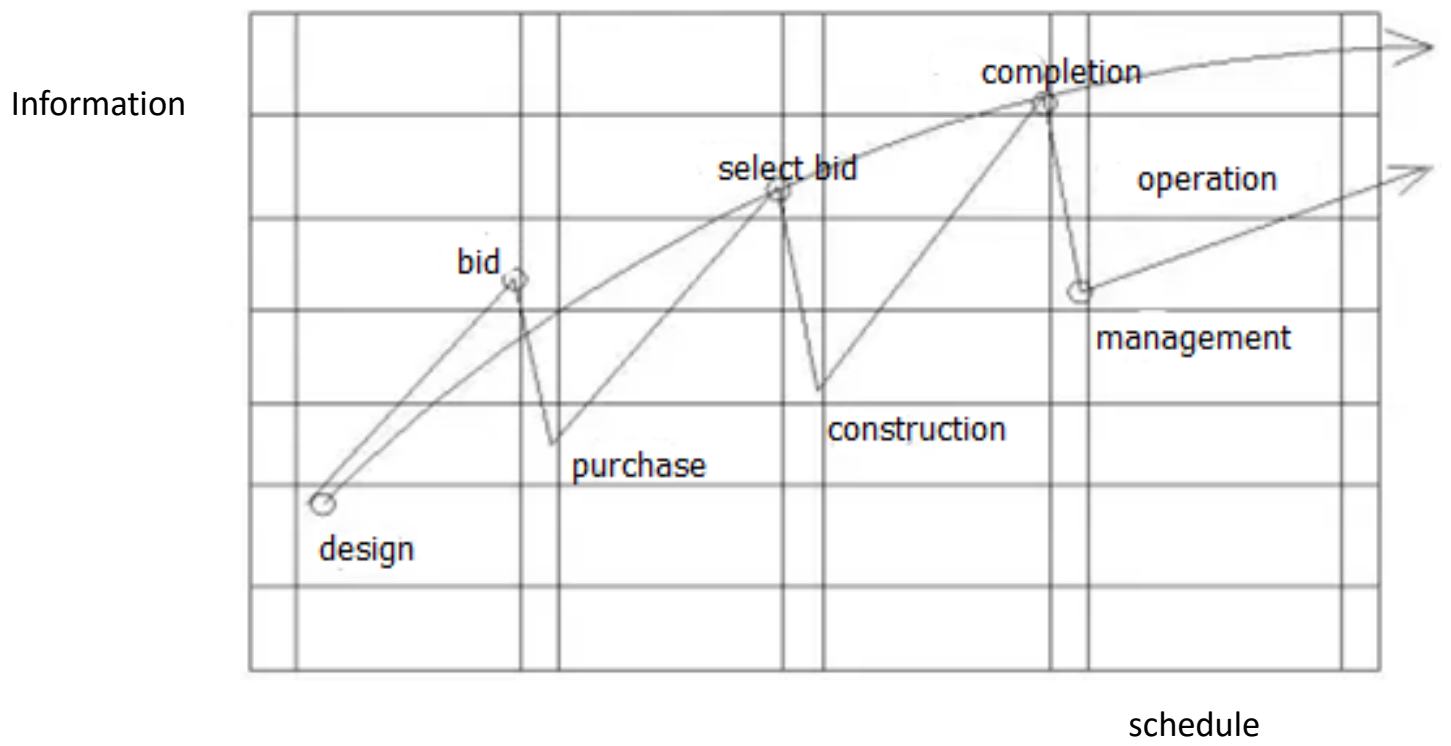

Figure 5. The loss of information resulted from the medium of paper[1]

In addition, according from the introduction of <introduction to construction projects information > written by Dr. Martin Fischl and Pittman Jon, we can find that paper media information transmission has resulted in the loss of information and the repeated collection of information, as shown in Fig. 5. In this graph, the above smooth curve represents the ideal information accumulation; the broken line represents the traditional information accumulation in the 
medium of paper. From the graph, we can see that architects and engineers have created and collected a large amount of information about the project in design phase including geometric information, functional information, environmental information, etc. To the bidding stage and construction stage, parts of information will be lost for the medium of paper. While, in order to accomplish project, construction contractors have to collect and study these lost information. Then the source of information may be various. However, the carrier of information becomes models which almost completely expressed the information collected by architects and engineers when BIM was adopted in project. Then, the source of the more parts of information that transmitted by model than that transmitted by paper become designers. That means the adoption of BIM changes the source of the same information in BIM project and traditional project. This is the other kind of influence of BIM on information source.

Impacts of BIM on transmitter (encoding). Information source encodes the wanted information into signal through transmitter. In fact, this process is equivalent to the information carried by model or by paper in construction project information exchange. For this process, in fact, the impact of BIM refers to the difference between information carried by paper and information carried by model.

BuildingSmart International definites building information model as the digital representation of physical and functional characteristics of a facility. As such it serves as a shared knowledge resource for information about a facility, forming a reliable basis for decisions during its life-cycle from inception onwards. From this definition, we can see that the first difference between these two form information is that one is digital information and the other is paper information. The second difference is about the content, including the amount of information and the type of information. Taking Revit information model as an example, a Revit model include model elements, view elements, and annotation elements. Model element data include basic data and ancillary data, basic data include geometric features, physical features, functional features, etc. Ancillary data include technical data, economic data, management data, etc. However, the ancillary data can't be expressed in drawings. In addition, the application of model makes it possible to design a lot of complex and difficult objects[7], thus increasing the amount of information of project as a whole.

Impacts of BIM on information channel. Channel refers to the medium that transmitted signal from one place to another. Most of the model-based digital information is transmitted through the network, and the paper-based information is mostly transmitted by mail. In comparison, the transmission speed of the former is faster than the latter. For the large amount information carried by model, compared with paper, model can transmit more information one time. As for the interference of "noise", both are subject to varying degrees of impact. We don't analyze it here.

Impacts of BIM on receiver (decoding). The decoding process is the process of turning the signal back into information. In fact, it is the inverse process of encoding process, and the impact of 'noise' makes it more complex than encoding.

Complete information includes grammatical information, semantic information and pragmatic information. However, in fact, the transmission of information is just the transfer of grammar information[8]. It is needed to rely on people's thinking or machine system if we want turn grammatical information back into complete information. Obviously, this process is completed by human in project management. So, compared with drawing, it is easier for people to reason out the complete information from model. Thus, the possibility of dissent and error can be reduced and we can reduce repeated requests for the same information[9].

Impacts of BIM on information sink. The sink is the recipient of information, as well the demander of information. The adoption of BIM has similar impacts on sink and source. That is new stakeholder would influence information sink. In addition, the application of BIM makes the preconstruction member ratio increased[10]; merchants of construction member will therefore receive more relevant information.

Impacts of BIM on feedback. Information feedback is the process that the receivers send the result of the application of information or the received information back to the source. The feedback process is actually the reverse flow of the feedback information. So this process experienced similar 
impacts as the normal flow of information.

\section{Conclusions and Limitations}

This paper gradually analyzed the impacts of BIM on information exchange between project stakeholders. In the end, we concluded that the adoption of BIM may produce new information source, create richer, more clear and accurate information, improve the efficiency of information transmission and reduce the possibility of wrong interpretation of information.

Impacts brought by the adoption of BIM may produce a series of effects. For example, the increased ratio of pre-construction member may make the supplier more important and change the relationship net among stakeholders; the adoption of BIM makes it possible to design a much more difficult object, which makes higher RFI in former stages and lower RFI in later stages[11], and changes the number of information exchange between ; the decrease of repeated collection of the same information improve production efficiency and many other effects like this[1].

While, this paper only discussed impacts of BIM on information exchange through literature analysis. It is lack of empirical support. Even though, we think that this paper can give project managers some advices to realize better management and to decide if they should adopt BIM.

\section{References}

[1] S. Ding, Introduction to information construction, China Building Industry Press, Beijing, 2005.

[2] M.P. Nepal, J.R.J. , a.A.A. Aibinu, Evaluations of BIM: Frameworks and Perspectives COMPUTING IN CIVIL AND BUILDING ENGINEERING, (2014).

[3] C. Lou, Basis of information management, Science Press, Beijing, 2005.

[4] H. Lasswell, The structure and function of communication in Sociology Communication University of China press, Beijing, 2012.

[5] W. Schram, Introduction to communication studies, Xinhua Press, Beijing, 1984.

[6] Y. Sun, Research on the whole life cycle information management of construction project based on BIM, in, Harbin Institute of Technology, 2011.

[7] D. Bryde, M. Broquetas, J.M. Volm, The project benefits of Building Information Modelling (BIM), International Journal of Project Management, 31 (2013) 971-980.

[8] B.Z. He, Lihou, Information management: Principle and method, Tsinghua University press, Beijing, 2006.

[9] K. Barlish, K. Sullivan, How to measure the benefits of BIM - A case study approach, Automation in Construction, 24 (2012) 149-159.

[10]K.-F. Chien, Z.-H. Wu, S.-C. Huang, Identifying and assessing critical risk factors for BIM projects: Empirical study, Automation in Construction, 45 (2014) 1-15.

[11]T. Hartmann, H. van Meerveld, N. Vossebeld, A. Adriaanse, Aligning building information model tools and construction management methods, Automation in Construction, 22 (2012) 605-613. 